\title{
Interference-Aware Cooperative Routing in Wireless Networks
}

\author{
Ido Ben-Tsion, Itzik Kitroser, Yehuda Ben-Shimol ${ }^{\star}$ \\ Communication Systems Engineering, Ben-Gurion University of the Negev
}

\begin{abstract}
We study interference-aware, many-to-many cooperative routing algorithms in wireless networks. Network performance is measured in terms of effective rate achieved when multiple frames are transmitted sequentially over a single cooperative path. Motivated by the overall network performance when multiple concurrent sessions are taken into consideration, we develop heuristic algorithms that reduce the interference caused by a session to a small geographic neighborhood. This allows the following frames and possibly the rest of the network to operate efficiently. We introduce an interference-aware performance metric based on the effective rate and evaluate the performance of several cooperative routing algorithms. Our heuristic algorithms show an improvement of $10-20 \%$ in the effective rate over traditional cooperative routing algorithms and an improvement of $10 \%$ over simple routing without cooperative transmission. We show that cooperation has little if any performance gain over simple relaying in terms of effective rate when inter-frame interference is not taken into consideration.
\end{abstract}

Index Terms-interference, cooperation, routing, wireless

\section{INTRODUCTION}

Cooperative transmission in wireless networks involves using relay nodes in an effective manner, taking advantage of the broadcast nature of the wireless channel. Several co-located relays receive and decode the same transmission, and later transmit it to the destination node or another relay group. This information is combined at each receiving node and may increase the signal to noise ratio (SNR) and, as a result, may increase the transmission rate.

Early research in cooperative transmission examined different relaying methods that allow cooperation, such as amplifyand-forward (AF), decode-and-forward (DF) and combinations thereof. Later, it became clear that cooperation would be most beneficial when applied only when information was correctly decoded. Another improvement suggested using a relay only if it improves performance.

Higher transmission rates may lead to increased network throughput, as long as the delay caused by the cooperation is tolerable. In spite of that benefit, using relay nodes consumes additional resources such as transmission power or frequency channels, and causes increased delay. Thus, there is an inherent trade-off between the cost of using a relay and the achievable rate while using the relay. A scenario where relay selection is required, arises when several nodes can serve as relays to a certain node. Furthermore, relay nodes having selfinformation, must delay their transmissions in order to take part in relaying information of other nodes. Scenarios such as those mentioned above require a decision on which node serves as a relay, and for which session the relay will transmit.
The diminishing benefit of larger groups of cooperating nodes led to several relay selection algorithms. Several cooperation algorithms are based on using the best relay out of a group of candidate nodes [1]-[3]. Another approach suggested organizing relays in relatively small cooperating groups [4], [5]. This approach has also reduced the search space, inspiring more complex heuristic algorithms. In this work, we develop efficient heuristic algorithms for finding a good transmission plan. Specifically, we require a transmission plan which achieves a maximum effective rate while imposing minimal interference and traffic delay.

A common assumption in cooperative transmission schemes restricts cooperation to 2-hop cooperation, connecting a source node with several relays, which in turn are connected to a single destination (e.g. [6]). A common modification (e.g. [7]) for this model allows several consecutive 2-hop cooperative links (i.e., links with multiple transmitters, as defined later). In this work, we assume a synchronized network, allowing a many-to-many cooperation scheme. The assumption of time synchronization can be justified by the availability of low cost GPS devices, which can be installed on each terminal [8]. This modification to the common model allows increased performance in terms of achievable rate, without sacrificing delay caused by longer paths with more hops. The performance gain is achieved with higher algorithm complexity, and calls for refined heuristic approaches.

Most recent research in this area focuses on finding a minimal-energy simple path or cooperative path between the source and the destination nodes. As the search space for the best relay assignment is exponential in the number of nodes in the network [4], [5], [9], [10], heuristic routing and cooperative routing algorithms based on simple paths have been suggested [1], [7], [9], [11], [12]. However, those algorithms are usually directed at cooperative paths which benefit a transmission of a single frame from source to destination. Instead, in this work we evaluate cooperative paths based on the effective transmission rate of a session, comprised of a sequence of frames or streams of information. The effective transmission rate is determined after eliminating the interference between sequential frames.

Little effort has been dedicated to optimizing the overall system performance of cooperative wireless networks. Since interference by nearby transmissions may reduce network efficiency [13], cooperative transmissions may have a negative impact on the overall system performance of cooperative wireless networks. Traditionally, research on cooperative transmissions does not account for the side-effects on the rest of the 
network [5], [9], or restricts cooperation to simple scenarios with negligible interference [1], [2], [11], thus reducing the potential benefits of cooperation.

Motivated by the overall network performance when multiple concurrent sessions are taken into consideration, we develop in this work heuristic algorithms that reduce the interference caused by a session to a small geographic neighborhood. This allows the following frames and possibly the rest of the network to operate efficiently. Our heuristic algorithms show a $10-20 \%$ improvement in the effective rate over traditional cooperative routing algorithms and a $10 \%$ improvement over simple routing without cooperative transmission.

The rest of this paper is organized as follows. Section II surveys related work in cooperative routing, related to the present work. In section III we describe system and network models. Section IV formulates the cooperative path problem followed by Section $\mathrm{V}$ which presents analyzes of some simple scenarios related to the problem. Section VI describes the heuristic cooperative routing algorithms. Section VII describes the simulation evaluating the performance of the algorithms and the results are presented in Section VIII. Conclusions and future work concludes the paper in Section IX.

\section{Previous WorK}

Cooperative relaying has been considered a promising technique in the quest to achieve optimal wireless network performance. In order to successfully incorporate cooperative relaying, several key issues need to be addressed. Relaying methods such as decode-and-forward (DF), amplify-and-forward (AF) and others were analyzed in [4], [14], in an attempt to categorize the scenarios in which each method achieves the capacity bounds. Simple relay selection algorithms based on channel quality estimation have been considered in [3], [4], [15].

Reducing the number of cooperating relays has been considered in various works. [4] observed the diminishing benefit of larger groups of cooperating terminals and suggested to partition the terminals into relatively small cooperating groups, in order to reduce the complexity of the search. [2] suggested selecting a single relay out of multiple candidates. [5] discussed a method for limiting the number of cooperating relays to a sequence of $L$ nodes along the path from source to destination, which was later improved in to the best $L$ nodes in the window of size $W$ along the path. An approach for limiting the relay search space based on network geometry was considered in [12], for simple routing algorithms without employing cooperative relaying.

The idea of constructing a cooperative-shortest-path (CSP), where the next hop in the cooperative path is determined based on the performance gained by cooperation, was studied in [7], [9], [11]. [9] implemented a Dijkstra based CSP algorithm, while [11] implemented a Bellman-Ford based algorithm. [7] also examined the performance gain of CSP vs. single-hop and simple multi-hop routing algorithms. They observed that using shorter hops is a preferred strategy, and as long as cooperation is used only when it can increase the rate, the achieved rate is comparable to that achieved by more complex algorithms.

\section{System AND NeTwORK MODEL}

We consider a managed multi-hop wireless network of $n$ nodes distributed over a given area. Nodes locations and channel gain coefficients are known to a central management unit. We assume that nodes are static or have low mobility. Each node transmits or receives using a single omni-directional antenna and half-duplex TDD mode is assumed. This means that each node can either transmit or receive at each time slot. Each node may serve as either a source, a destination, or a relay at a given time slot. We assume that nodes can buffer transmissions up to $B$ time slots.

Nodes may cooperate in transmitting and receiving information using Decode-and-Forward relaying method, although other methods may be adequate as well. We assume a synchronized network, where correlated signals can be combined at receiving nodes for effective cooperation (i.e., improving the SNR at the receiver).

For simplicity, all nodes transmit using the same power $P$ (however, the rate expressions can be extended to allow for individual power levels for nodes). Unicast sessions are assumed, resulting with a single destination for each source. In this work we assume a single active session at a time, comprised of a single source-destination pair.

Our network model resembles that of [6], with few changes that allow many-to-many cooperative links. The network $G=$ $(V, L, W)$ is defined by the set of nodes $V$, the set of links $L$ and a weight function $W: L \rightarrow \mathbb{R}^{+}$. The links are either cooperative or direct (non-cooperative). The weights on the links correspond to the SNR or CSNR (Cooperative SNR, defined below) for each direct or cooperative link, respectively. The weight function $W$ depends on the transmission power $P$, the channel gain matrix $H$ (constructed from measured statistical values) and the noise (for simplicity, unit variance noise is assumed). The channel gain matrix $H=\left[\sqrt{h_{u v}}\right]$ represents the corresponding channel gain coefficients for each pair of nodes.

In the following expressions, a small letter denotes a single node, and a capital letter is used to refer to a set of nodes. Using this notation, the following definitions for SNR (oneto-one $i \rightarrow j$ and one-to-many $i \rightarrow J$ ) and CSNR (many-to-one $I \rightarrow j$ and many-to-many $I \rightarrow J$ ) were derived from expressions given in [6], [16]:

$$
\begin{gathered}
w_{i j}=h_{i j} P \\
w_{i J}=\min _{j \in J} w_{i j} \\
w_{I j}=\left(\sum_{i \in I} \sqrt{h_{i j}}\right)^{2} P \\
w_{I J}=\min _{j \in J} w_{I j}
\end{gathered}
$$

Several definitions and notations are needed to define cooperative communication. The requirement for transmitting $q$ frames from origin node $o$ to destination node $d$ is denoted by a session $Q(o, d, q)$. A cooperation group (CG) is a set 
of nodes which, at a certain time slot, either receive or transmit the same frame. The size of a CG is defined as the number of nodes in the $C G$ and is limited to $C_{\max } \in \mathbb{N}$. Since we are dealing with unicast transmissions, we denote by $\mathrm{CG}_{o}=\{o\}$ and $\mathrm{CG}_{d}=\{d\}$, the origin $\mathrm{CG}$ and the destination $\mathrm{CG}$, respectively. At any given time slot, each node is allowed to participate only in a single CG.

A cooperative link $\mathrm{CL}_{I J}$ connects a source $\mathrm{CG} I$ to a target CG $J$ iff

$$
w_{I J} \geq w_{\min }
$$

where $w_{\min }$ is the minimal SNR supporting a transmission at some minimal rate with some outage and error probabilities. The set of CLs in the network is defined as

$$
L=\left\{(I, J) \mid I, J \subset V \wedge w_{I J} \geq w_{\min }\right\}
$$

where $I, J$ are CGs. Note that CLs are not necessarily an aggregation of direct links, since in some cases a direct link does not exist and a CL may allow connectivity (see example in Figure 1).

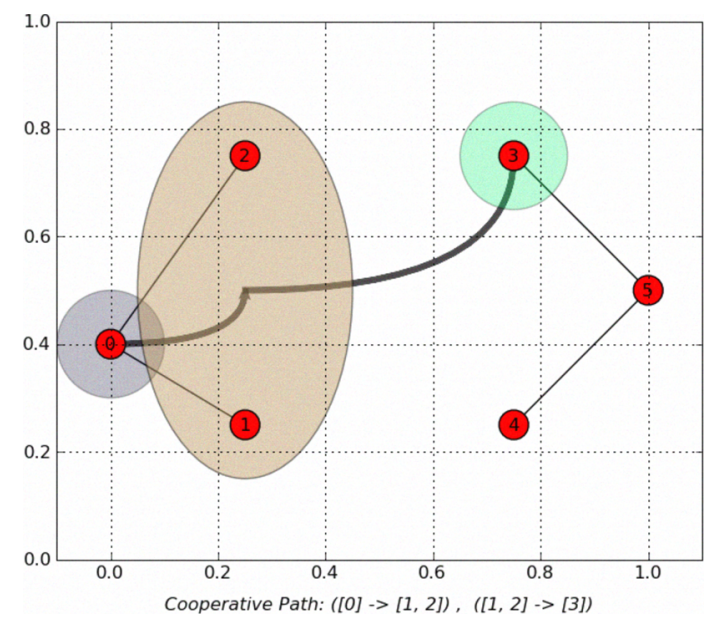

Figure 1: A wireless network connected only when cooperation is allowed. Straight lines represent direct links while curved lines represent cooperative links. This figure depicts a cooperative path between origin 0 and destination 3 . Note that a direct link to node 3 does not exist while a cooperative link does.

At time slot $k$, frame $f$ is transmitted over a single CL $\left(S^{f}(k), T^{f}(k)\right) . S^{f}(k)$ and $T^{f}(k)$ denote the source CG and the target CG of frame $f$ at time slot $k$, respectively. This means that every node in $S^{f}(k)$ transmits the same copy of frame $f$ and every node in $T^{f}(k)$ is receiving the same copy of frame $f$. We assume that only traffic that was decoded successfully by a source CG can be sent over a CL to a target CG.

A cooperative path (CP) is a set of CLs, $\mathrm{CP}=$ $\left\{\mathrm{CL}_{1}, \mathrm{CL}_{2}, \ldots, \mathrm{CL}_{m}\right\}$, such that each $\mathrm{CL}_{i}$ connects $\mathrm{CG}_{i}$ and $\mathrm{CG}_{i+1}$ (i.e., $\mathrm{CG}_{i}$ is the source $\mathrm{CG}$ and $\mathrm{CG}_{i+1}$ is the target CG). The length $|\mathrm{CP}|=m$ is defined as the number of CLs in the $\mathrm{CP}$. The maximal rate of a $\mathrm{CP}, R(\mathrm{CP})$, is the rate of the lowest capacity $\mathrm{CL}$ in the path (cut-set bound):

$$
R(\mathrm{CP}) \leq \min _{i \in\{1, \ldots,|\mathrm{CP}|\}} \log \left(1+w_{i}\right)
$$

where $w_{i}$ denotes the CSNR of $\mathrm{CL}_{i}$. We denote by $\mathrm{CP}(Q)$ the cooperative path used by the session $Q$. The delay imposed by a $\mathrm{CP}$ is the number of time slots used until the first frame reaches the destination, and is equal to $|\mathrm{CP}|$.

A transmission plan (TP) is an action vector - assignment of actions (transmit_frame_f/receive/idle) for nodes - at a specific time slot $k$ :

$$
\mathrm{TP}_{k}=\left(a_{1}, \ldots, a_{n}\right) \quad a_{u} \in\left\{t^{f}, r, i\right\}
$$

where $t^{f}$ denotes transmitting the $f$-th frame, which was stored at a previous time slot in the range $[k-B, k-1]$.

Cooperative communication is realized via a sequence of transmission plans. A transmission plan sequence (TPS) is defined as:

$$
\mathrm{TPS}_{i j}=\left(\mathrm{TP}_{i}, \ldots, \mathrm{TP}_{k}, \ldots, \mathrm{TP}_{j}\right) \quad i \leq k \leq j
$$

The length of a TPS is defined as the number of TPs (number of time slots) in the sequence: $\left|\mathrm{TPS}_{i j}\right|=j-i+1$. We denote by TPS $(Q, \mathrm{CP})$ the TPS used to transmit the session $Q$ over a CP. A valid TPS requires that every frame $f$ is transmitted over at most a single CL, $\mathrm{CL}^{f}(k)=\left(S^{f}(k), T^{f}(k)\right)$, such that no two frames interfere with each other.

Frame $f_{i}$ interferes with the transmission of frame $f_{j}$ $\left(f_{i}<f_{j}\right.$, i.e., $f_{i}$ was transmitted before $f_{j}$ ) at time slot $k$ if there exists a CL of the form $(X, Y) \in L$, such that $X \subseteq S^{f_{j}}(k)$ and $Y \subseteq T^{f_{i}}(k)$ (i.e., CG $X$ transmits frame $f_{j}$ and the CG $Y$ receives both frame $f_{i}$ and frame $f_{j}$ ). A special case of interference is the half-duplex constraint, where $X \cap Y \neq \emptyset$. Therefore, we define an interference indicator function $I$ as follows:

$$
I(\operatorname{TPS}(Q, C P))=\left\{\begin{array}{cc}
1 & \exists k: \mathrm{CL}\left(S^{f_{i}}(k), T^{f_{j}}(k)\right) \in \mathrm{CP} \wedge \\
& \exists(X, Y) \in L: X \subseteq S^{f_{j}}(k) \wedge \\
& Y \subseteq T^{f_{i}}(k) \\
0 & \text { otherwise }
\end{array}\right.
$$

\section{Problem Statement}

The effective rate for a given session $Q, \operatorname{ER}(Q)$, is defined as the achievable transmission without inter-frame interference:

$$
\mathrm{ER}(Q, \mathrm{CP}(Q))=\left\{\begin{array}{cc}
R(\mathrm{CP}) / h(\mathrm{CP}) & q>1 \\
R(\mathrm{CP}) & q=1
\end{array}\right.
$$

where $h(\mathrm{CP})=|\operatorname{TPS}(Q, \mathrm{CP})|-q+1$ is the minimum number of time slots between consecutive frames $f_{i}$ and $f_{j}$, such that $f_{i}<f_{j}$ and $I(\operatorname{TPS}(Q, C P))=0$. As a worst case scenario, which is more frequent as the number of hops in the $\mathrm{CP}$ decreases, $h(\mathrm{CP})=|\mathrm{CP}|$.

For a given session $Q(s, d, q)$, we require a $\mathrm{CP}$ (and a $\operatorname{TPS}(Q, \mathrm{CP}))$ that maximizes $\operatorname{ER}(Q, \mathrm{CP}(Q))$ :

$$
\max _{\mathrm{CP}(Q)} \operatorname{ER}(Q, \mathrm{CP}(Q))
$$




$$
\text { s.t. }|\mathrm{CP}(Q)| \leq \tau_{\max }
$$

where $\tau_{\max }$ is the maximal delay allowed for session $Q . \tau_{\max }$ is represented as the number of time slots until the first frame reaches the destination node.

Algorithms for finding CPs may return several solutions all having the same maximal ER and all are preserving the constraints for $\tau_{\max }$. We further differentiate between those solutions on the basis of their "environmental effect". Therefore we define an interference-aware performance metric for $\mathrm{CP}$ efficiency based on the effective rate - Interference-AreaAware-Effective-Rate (IAAER) as follows:

$$
\begin{gathered}
\operatorname{IAAER}(Q, \mathrm{CP})=\mathrm{ER}(Q, \mathrm{CP}(Q)) / \Delta_{A} \\
\Delta_{A}=\frac{1}{|\mathrm{CP}|} \cdot \frac{1}{A} \cdot \sum_{i=1}^{|\mathrm{CP}|} a_{i}
\end{gathered}
$$

where $a_{i}$ is the area affected by the transmissions on $\mathrm{CL}_{i}$ and $A$ is the area affected by the transmissions in the entire network. IAAER is introduced with overall network performance in mind, to examine the CP's effect on the rest of the network, when there are multiple simultaneous sessions. The actual shape and area of $a_{i}$ usually depend on random channel parameters and are quite complex. However a computational effective approximation to $a_{i}$ can be obtained by a convex hull of circles surrounding the nodes in the source CG (see Figure (2)), each with a radius of $r=d_{0} \cdot\left(P / w_{\min }\right)^{1 / \alpha}$ or similar expressions based on different path-loss models. Using this approximation the area can be calculated as an area of a convex polygon. Note that when the session $Q$ includes multiple frames, IAAER $(Q, \mathrm{CP}) \sim R(\mathrm{CP}) / \sum_{i=1}^{|\mathrm{CP}|} a_{i}$.

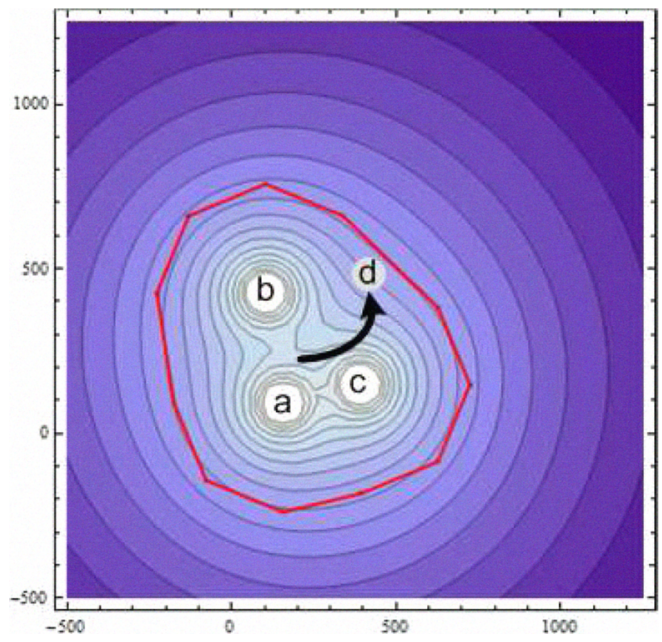

Figure 2: An example of approximating the Interference-Area of a CG having 3 nodes $\{a, b, c\}$ transmitting to a single destination node $d$

\section{ANAlysis of Simple ScEnarios}

Figure 3 shows some simple scenarios, which help to examine the benefit of cooperative transmission in terms of effective rate. In the following figures, straight lines represent direct links while curved lines represent cooperative links. Link weights denote the link capacity.

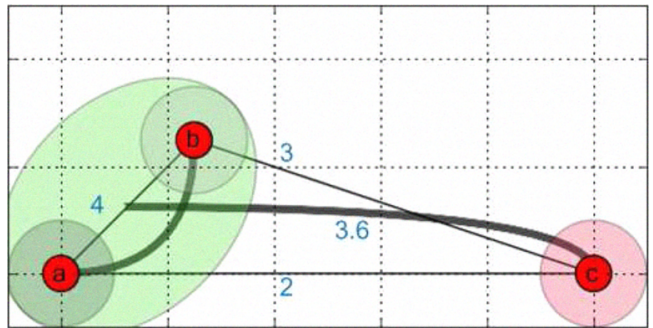

(a) Three node network

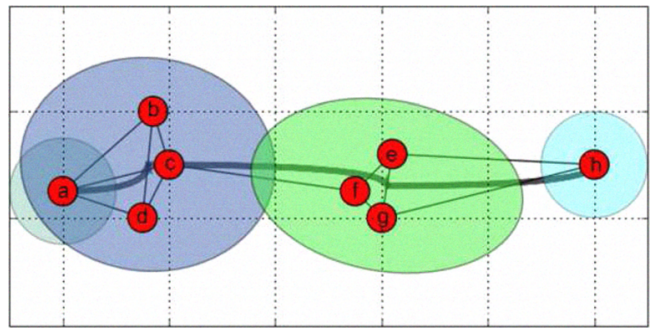

(b) Clusters

Figure 3: Simple scenarios

A. Three node network (single source, single relay, single destination)

In the basic cooperation scenario, illustrated in Figure 3a, the origin node $a$ may use relay $b$ in order to increase the rate at which it can transmit to destination $c$ from $2 \mathrm{bps} / \mathrm{Hz}$ to $3 \mathrm{bps} / \mathrm{Hz}$. When both $a$ and $b$ cooperate, this rate increases to $\sqrt{2^{2}+3^{2}} \cong 3.6 \mathrm{bps} / \mathrm{Hz}$. However, when multiple frames are taken into consideration, both TPSs described suffer a penalty of 2 hops, reducing their ER to $1.5 \mathrm{bps} / \mathrm{Hz}$ and 1.8 $\mathrm{bps} / \mathrm{Hz}$, respectively. The maximal effective rate at which $a$ can transmit to $c$ is therefore $2 \mathrm{bps} / \mathrm{Hz}$, achieved by a direct transmission from $a$ to $c$.

\section{B. Clusters}

A scenario demonstrating the potential benefits of expanding the relay search area beyond the optimal noncooperative path and applying many-to-many cooperation is shown in Figure 3b. Origin node $a$ and destination node $h$ are connected by a maximum capacity simple path $(a, d, c, f, e, h)$. The nodes $b, c, d$ are clustered over a relatively small area, as are nodes $e, f, g$. A manyto-one cooperative routing algorithm may choose the CP $((\{a\},\{b, c, d\}),(\{b, c, d\},\{f\}),(\{f\},\{e, g\}),(\{e, f, g\},\{h\}))$, which requires 4 time slots. Since the nodes $e, f, g$ are clustered, the CLs $(\{b, c, d\},\{e\}), \quad(\{b, c, d\},\{f\})$ and $(\{b, c, d\},\{g\})$ have similar capacities and may be effectively combined by many-to-many cooperation to a single $\mathrm{CL} \quad(\{b, c, d\},\{e, f, g\})$, without an additional transmission. The chosen $\mathrm{CP}$ is therefore $((\{a\},\{b, c, d\}),(\{b, c, d\},\{e, f, g\}),(\{e, f, g\},\{h\}))$, requiring only 3 time slots, and the shorter CP may increase the effective rate. 


\section{Heuristic Algorithms}

The main ideas behind our interference-aware heuristic cooperative routing algorithms is to limit the geographical area affected by transmissions and to limit the maximal size of CGs to $\rho \in \mathbb{N}$. Both have the positive effects of limiting the relay search space and reducing overhead of CGs maintenance in the process. In the following we present two algorithms for calculating CLs and CPs. The Greedy-Route-Improvement (GRI) algorithm is a greedy algorithm that is based on a simple (noncooperative) route from origin $o$ to destination $d$, upon which the weakest $\mathrm{CL}$ is repeatedly improved by adding cooperation. The second algorithm - Dynamic Selection (DPS) - combines routing and cooperation, constructing a $\mathrm{CP}$ recursively by connecting shorter CPs. A detailed description, analysis and results for both algorithms follows.

\section{A. Greedy-Route-Improvement - GRI}

First, the shortest simple path route (spr) from $o$ to $d$ (maximum capacity route) is constructed, using a max-flow algorithm similar to the Prim-Dijkstra algorithm. Each node is considered as a CG composed of a single node. Next, we look for the bottleneck CL in the path - say $\mathrm{CL}_{i}$, and attempt to increase its capacity by adding relays. GRI looks for a relay $r$, whose addition to the source $\mathrm{CG}$ of $\mathrm{CL}_{i}$ would maximally increase its capacity, while minimizing the degradation in capacity of the previous CL, which changes as well. The search for the relay is therefore limited to a circle with radius $d_{\text {coop }}$ around $\mathrm{CG}_{i-1}$.

If the first link is a bottleneck, then there is no way to increase the capacity of the $\mathrm{CP}$ by cooperation, and the current $\mathrm{CP}$ is used. Otherwise, as long as the CG size limit is not reached, we add the relay $r$ and improve the link. This procedure continues until no further improvement is possible. We repeat this process for each sub-route of the simple route, allowing the discovery of shorter CPs which would increase the ER.

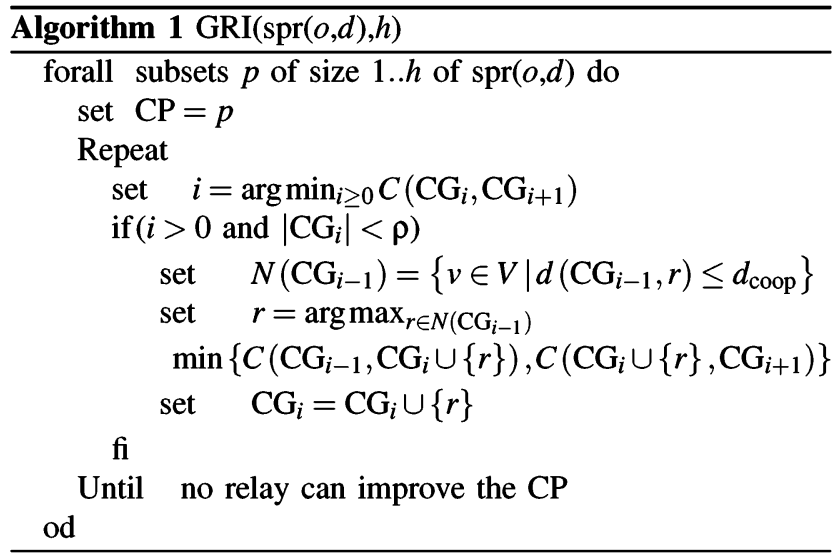

\section{B. Dynamic Path Selection - DPS}

DPS aims at finding the best CP in the restricted searchspace, by finding the maximal capacity $\mathrm{CP}$ using 1 to $h_{\max }$ hops (CLs), where each hop introduces another CG as a relay group. DPS uses a method similar ${ }^{1}$ to Dynamic Programming, with a memoization structure $C(I, K, h)$, which stores the capacity of the best CP between source CG $I$ and target CG $K$ in $h$ hops, and is defined as:

$C(I, K, h)= \begin{cases}\max \{C(I, K, h-1), & h>1 \\ \left.\min _{J \subseteq N(I)}\{C(I, J, 1), C(J, K, h-1)\}\right\} & \\ \text { Capacity }(I, K) & h=1\end{cases}$

where $I, J, K$ are CGs restricted to a maximal number of nodes $\rho, N(I)=\left\{r \in V \mid d(I, r) \leq d_{\text {coop }}\right\}$ is the group of geographic neighbors of the CG $I$ and $d(I, r)=\max _{i \in I}\|i-r\|$ is the euclidean distance between the group $I$ and the relay $r$. The maximal capacity $\mathrm{CP}$ is therefore

$$
\mathrm{CP}=\max _{\mathrm{CP}, h} C(\{o\},\{d\}, h)
$$

The maximal number of hops $h$, is determined by $h=$ $\min \{\tau, \max \{\lceil\sqrt{N}\rceil,|\mathrm{SPR}|\}\}$, where SPR is a simple route from $o$ to $d, \sqrt{N}$ is the diameter of the network (assuming a uniform distribution of nodes) and $\tau$ is a maximum delay constant (i.e., $\tau \leq 8$ ). In order to prevent dead-ends when $J \subseteq N(I)=I$ (due to the reduced search area), we expand the search to $J \subseteq N(x)$ where $x$ are relay nodes in a simple (noncooperative) route from $o$ to $d$. A variation over this method is to maximize the normalized-capacity.

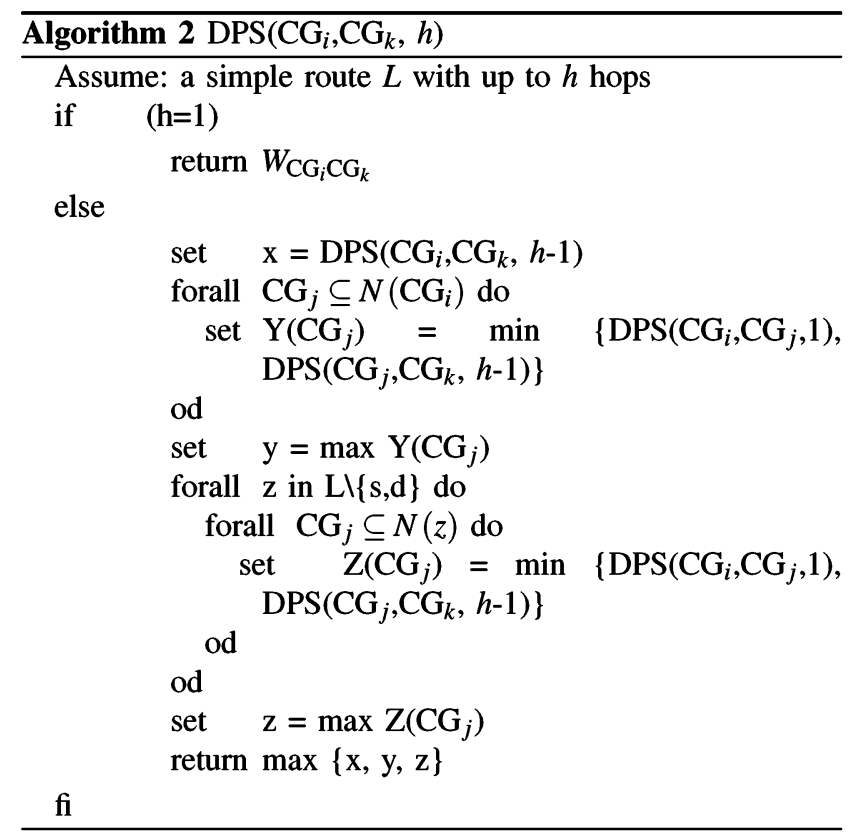

\section{Details and Analysis}

In GRI, the construction of the simple route from $o$ to $d$ may affect the quality of the $\mathrm{CP}$ - each selected relay is kept during all further improvements. If a relay with poor performance is selected, it might take the place of better relays which could have been used. DPS solves this problem by considering CPs based on all combinations of relay groups. DPS may use

\footnotetext{
${ }^{1} \mathrm{DP}$ requires an optimal sub-structure of the solution, a condition which is not satisfied when our goal is the ER of the CP, due to the dependency between the combined CPs.
} 
Cap

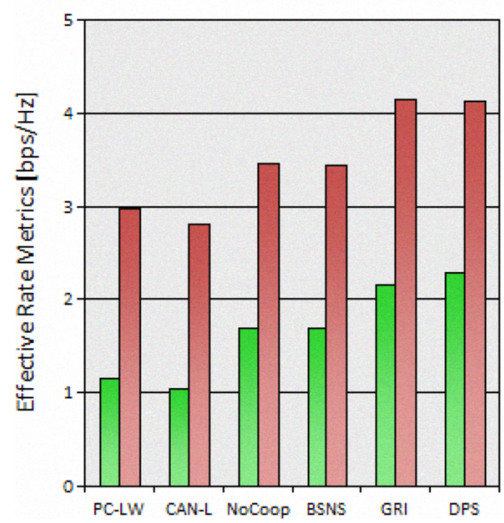

Greedy

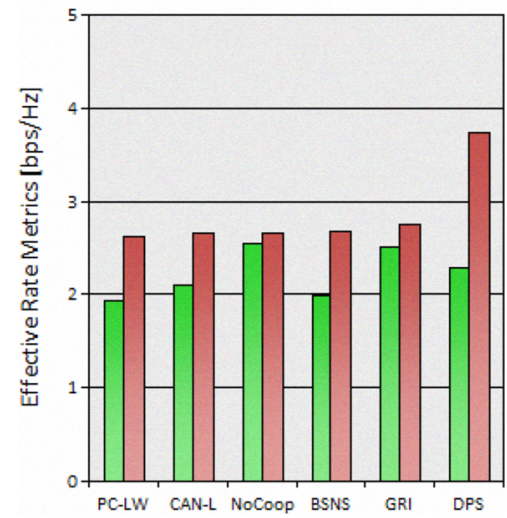

NCap

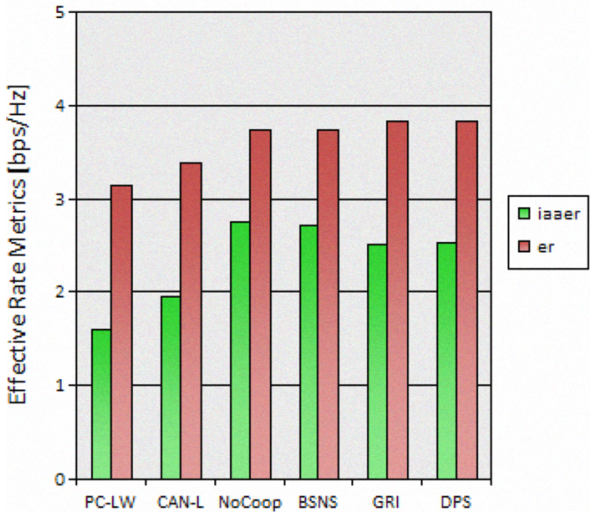

Figure 4: Effective Rate metrics comparison, when GRI and DPS use a relay search area of $\frac{1}{3} W$ and a maximum CG size of 3

the simple route, but also searches for a different path that might improves the performance. In order to achieve that, DPS introduces higher complexity and is much more intensive in computation.

GRI and DPS are affected by the choice of $d_{\text {coop }}$, which limits the geographical area for searching relay nodes. The complexity of the relay search is therefore a function of the coverage area size, the node density and $d_{\text {coop. }}$ Both GRI and DPS select the CP with highest effective rate from a candidate CP set. In GRI, the candidate CPs are formed of sub-paths of the simple route, where in DPS, candidates are the maximal effective rate paths of different lengths.

The complexities of GRI and DPS are presented here without proof. The complexity of GRI is $O((m+n) \log n+h ! \cdot \rho h M)$, where $h$ is the number of hops in the route, $\rho$ is the maximal size of a CG and $M$ is the number of nodes in the restricted search area. The complexity of DPS is dominated by the number of slots in the memoization structure $C(I, K, h)$, which is $O\left(h \cdot 4^{M}\right)$.

GRI optimizes the ER by selecting the CP which maximizes the ER among a larger set of $O(h$ !) sub-routes, while DPS optimizes the capacity (Cap) or the normalized capacity (NCap) and selects the CP which maximizes ER only among $h$ CPs, discriminating different length CPs based on the ER. As a result, GRI's performance may, on some occasions, surpass DPS's performance.

\section{Simulation}

In order to evaluate our heuristic cooperative routing algorithms, we generated networks of various sizes, $N \in$ $\{6, \ldots, 20\}$ nodes, chosen uniformly in a square coverage area of width $W=0.3 * \sqrt{N} \mathrm{~km}$, in order to keep the average node density constant. In each network, a pair of nodes are randomly selected as source and destination. Routing is trivial when a direct transmission from source to destination is feasible, therefore we chose relatively distant nodes by selecting a source node $x$ uniformly and selecting its destination $y$ from a distance-based distribution $P(Y=y)=\frac{d(x, y)}{\sum_{z} d(x, z)}$. We also filtered out trivial results, where direct links were the best choice.
Our path loss model is of an Urban Microcell LOS with $2.4 \mathrm{GHz}$ carrier frequency. Channels are reciprocal, with random i.i.d. shadowing factors generated from a LogNormal distribution with $0 \mathrm{~dB}$ mean and $8 \mathrm{~dB}$ variance, and a Rayleigh fading with $8 \mathrm{~dB}$ variance. The transmitter powers are set to $P=0.1 \mathrm{~W}$. We also assume receiver noise of $7 \mathrm{~dB}$.

We compared the performance of our heuristic cooperative routing algorithms to simple relaying without cooperation (denoted as NoCoop) and to an adapted version of several cooperative routing algorithms given in the literature: Cooperation-Along-Non-cooperative-path (CAN-L [5]), Progressive-Cooperation (PC-LW [5]), and Best-Select-in-theNeighbor-Set (BSNS [1]) which was modified with smartcooperation [7] to cooperate only when the capacity of the CL increases.

The target function of the algorithms was modified from minimal power based on constant rate to maximum capacity based on constant power. We further modified each cooperative routing algorithm to use different simple-path-routing (SPR) algorithms and extend them to a cooperative path. The SPRs used are greedy-route (denoted as Greedy), maximal-capacityroute (denoted as Cap) and maximal-normalized-capacityroute (denoted as NCap). In Greedy, the next hop node is chosen based on maximal SNR to the destination. Cap is based on a Dijkstra algorithm and maximizes the minimal capacity link in the path. NCap is similar to Cap, but maximizes the minimal capacity divided by the number of hops.

In each of the algorithms which allow for more than one relay in a CG, we evaluated its performance while limiting the maximum number of relays to $\rho \in\{2, \ldots, 5\}$. The geographic limiting parameter in GRI and DPS, $d_{\text {coop }}$, was selected as a factor of the coverage area width, such that $d_{\text {coop }} \in\left\{W, \frac{1}{2} W, \frac{1}{3} W, \frac{1}{4} W\right\}$.

\section{RESULTS}

Figures 4-6 illustrate the main results of this work.

Figure 4 compares the effective rate (ER, illustrated by the right bar) and the interference-aware metric IAAER (illustrated by the left bar) for each algorithm, based on different simple 


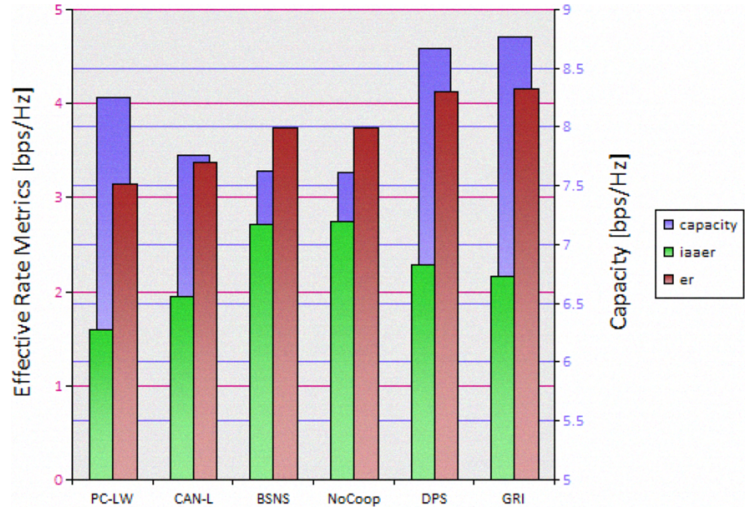

Figure 5: Effective Rate metrics and CL capacity comparison, based on the best choice of SPR algorithm, with the parameters $G R I-3-\frac{1}{3} W, D P S-3-\frac{1}{3} W, C A N-3, P C-3-2$

path routing algorithms. The results on the left chart are based on Capacity maximizing SPR, those in the middle are based on Greedy SPR, and those on the right chart are based on Normalized Capacity maximizing SPR.

Cooperation is most beneficial when the basic routing scheme is unaware of interference (Greedy, Cap). When NCap is used as SPR, the ER gain from cooperation is negligible. Nonetheless, the maximal ER is gained by GRI and DPS when used with Cap SPR, as they allow larger CL capacity with only a slight degradation in performance due to interference. DPS may use the path offered by the SPR, but searches for a different path that might improve the performance, and therefore is mainly unaffected by the choice of SPR. GRI searches for sub-paths based on the SPR and improves them by cooperation and is therefore more affected by the choice of SPR. GRI's performance is usually comparable to that of DPS and, as explained earlier, may even surpass DPS's performance on some occasions. On our simulation, the relay search area used by DPS was restricted in order to reduce the computational complexity in larger networks. As a result, the average performance of GRI in terms of ER was sometimes better than that of DPS. However, this increase in ER was achieved with higher interference, as illustrated by the IAAER metric.

Figure 5 compares the ER, IAEER and the bottleneck CL capacity (illustrated by the blue bar on the back of the chart) for each algorithm, using the best choice of SPR algorithm, that is, the one which maximizes the session ER. This figure allows us to compare the potential performance of the different approaches discussed in this work, each with its own optimal parameters. Even though the bottleneck CL capacity increases with the addition of relays (CANL, PCLW), the increased interference to other frames in the same CP (and possibly the rest of the network) reduces the ER. Cooperation in such cases has little, if any, performance gain over simple relaying (NoCoop). One strategy to combat this interference is to limit cooperation to a single relay (BSNS). However, taking this interference into account and using cooperative relays in a limited region (GRI, DPS) improves performance over simple relaying.

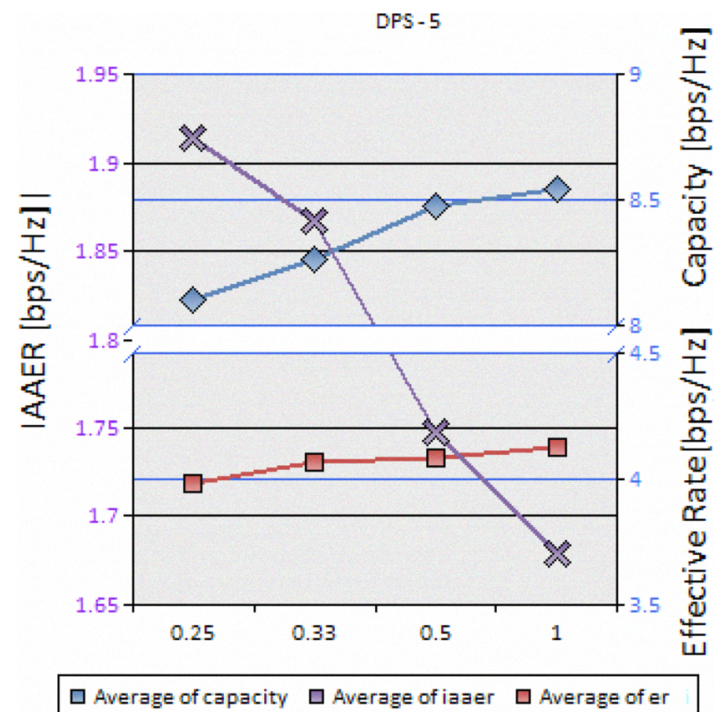

Figure 6: Capacity and ER with DPS-5-NCap and different relay search areas, defined by $d_{\text {coop }} \in\left\{W, \frac{1}{2} W, \frac{1}{3} W, \frac{1}{4} W\right\}$

Figure 6 compares the ER metrics and the bottleneck CL capacity of DPS-5-NCap, based on varying size of relay search area. The left scale depicts the IAAER and is continuous. The right scale is split between ER on the bottom part of the chart and the Capacity on the top one. Increasing the search area for relays increases the capacity and the ER, but as a side-effect also increases the interference area, as can be seen by the decrease in the IAAER metric. This figure depicts the idea behind limiting the relay search to a relatively small area, resulting in comparable ER while causing only a small interference. This trait will have even higher impact when multiple simultaneous sessions are taken into consideration.

\section{CONClusions And Future Work}

Interference-aware routing or cooperative routing algorithm have the potential to increased network performance, and this served as a motivation for the present work. In this work we discuss cooperation based routing and measure its performance in cases where delay and "environmental disturbance" are of importance. We find that cooperation has little, if any, performance gain over simple relaying in terms of effective rate when inter-frame interference is not taken into consideration while computing the $\mathrm{CP}$. Also, using effective rate as a measure we show that increasing the minimal capacity along the cooperative path does not guarantee that the resulting $\mathrm{CP}$ is of superior performance. We also showed that CPs having similar performance need to be further discriminated according to their effect on the surrounding nodes. This metric is important for cases where multiple simultaneous sessions need to use the same network, using cooperation. For that purpose we presented a new performance metric, namely, InterferenceArea-Aware-Effective-Rate, which is used to compare the performance of various algorithms for CP calculations. We 
also showed that searching for multiple relays in a relatively small area may lead to higher performance in terms of effective rate, while causing only limited interference to the rest of the network.

An interesting result is that, while our algorithms allow for larger cooperation groups, those actually selected were relatively small. The intuition behind this result is that limiting the number of cooperating relays has positive effects on a sequence of transmissions (multiple frames) and on the rest of the network, due to the half-duplex constraint and the interference. An algorithm that allows varying number of relay nodes, depending on the local environment at the bottleneck link, performs better on average than algorithms that use a constant number of relays, whether small or large. Larger groups of relays should only be used to bridge over a gap caused by the sparsity of nodes distribution. We also find that on dense networks, the use of cooperation seldom has any benefit on performance.

For future work, we plan on improving the heuristic algorithms to further account for interference during the construction of the cooperative path. This will improve both the effective rate of a single $\mathrm{CP}$ and the overall network effective rate, when multiple source destination pairs are considered. Further improvement to network performance may be gained by simultaneous optimization of several sessions, each using a $\mathrm{CP}$ which does not degrade the overall network effective rate.

\section{REFERENCES}

[1] J. Luo, R. Blum, L. Greenstein, L. Cimini, and A. Haimovich, "New approaches for cooperative use of multiple antennas in ad hoc wireless networks," in Vehicular Technology Conference, 2004. VTC2004-Fall. 2004 IEEE 60th, vol. 4, 2004.

[2] E. Beres and R. Adve, "On Selection Cooperation in Distributed Networks," in Conference on Information Sciences and Systems (CISS 2006), pp. 1056-1061, 2006.

[3] A. Nosratinia and T. Hunter, "Grouping and partner selection in cooperative wireless networks," IEEE Journal on Selected Areas in Communications, vol. 25, no. 2, pp. 369-378, 2007.

[4] J. Laneman, Cooperative Diversity in Wireless Networks: Algorithms and Architectures. PhD thesis, Massachusetts Institute of Technology, 2002.

[5] A. Khandani, J. Abounadi, E. Modiano, and L. Zheng, "Cooperative Routing in Wireless Networks," in Proceedings of the Annual Allerton Conference on Communication Control and Computing, vol. 41, pp. 1270-1279, Springer, 2003.

[6] E. Yeh and R. Berry, "Throughput Optimal Control of Cooperative Relay Networks," IEEE Transactions on Information Theory, vol. 53, no. 10, pp. 3827-3833, 2007.

[7] E. Beres and R. Adve, "Cooperation and Routing in Multi-Hop Networks," in IEEE International Conference on Communications, 2007. ICC'07., pp. 4767-4772, 2007.

[8] J. Elson, "Time synchronization in wireless sensor networks," tech. rep., May 31, 2003.

[9] F. Li, K. Wu, and A. Lippman, "Energy-efficient cooperative routing in multi-hop wireless ad hoc networks," in Performance, Computing, and Communications Conference, 2006. IPCCC 2006. 25th IEEE International, p. 8, 2006.

[10] E. Beres and R. Adve, "Selection Cooperation in Multi-Source Cooperative Networks," IEEE Transactions on Wireless Communications, vol. 7, no. 1 , pp. 118-127, 2008.

[11] A. Ibrahim, Z. Han, and K. Liu, "Distributed Energy-Efficient Cooperative Routing in Wireless Networks," in Global Telecommunications Conference, 2007. GLOBECOM'07. IEEE, pp. 4413-4418, 2007.

[12] D. Chen, M. Haenggi, and J. Laneman, "Distributed Spectrum-Efficient Routing Algorithms in Wireless Networks," in Information Sciences and Systems, 2007. CISS'07. 41st Annual Conference on, pp. 649-654, 2007.
[13] P. Gupta and P. Kumar, "The capacity of wireless networks," IEEE Transactions on Information Theory, vol. 46, no. 2, pp. 388-404, 2000.

[14] A. Sendonaris, E. Erkip, B. Aazhang, Q. Inc, and C. Campbell, "User cooperation diversity. Part I. System description," IEEE Transactions on Communications, vol. 51, no. 11, pp. 1927-1938, 2003.

[15] J. Laneman, D. Tse, and G. Wornell, "Cooperative diversity in wireless networks: Efficient protocols and outage behavior," IEEE Transactions on Information Theory, vol. 50, no. 12, pp. 3062-3080, 2004.

[16] L. Xie and P. Kumar, "A network information theory for wireless communication: scaling laws and optimal operation," IEEE Transactions on Information Theory, vol. 50, no. 5, pp. 748-767, 2004. 